\title{
A SiPM-based VLC Receiver for 3.45 Gigabits/s Communication Using OOK Modulation
}

\author{
William Matthews, Zubair Ahmed, Wajahat Ali and Steve Collins \\ Department of Engineering Science \\ University of Oxford \\ Oxford, United Kingdom \\ ORCID: 0000-0002-2388-4369, 0000-0003-4556-6158, 0000-0002-6511-5015, 0000-0002-3874-8319
}

\begin{abstract}
Results are presented which show that by using a SiPM with a higher maximum output pulse rate it is possible to increase the highest maximum OOK data rate ever achieved at a BER of $10^{-3}$ from $2.4 \mathrm{Gbits} / \mathrm{s}$ to $3.45 \mathrm{Gbits} / \mathrm{s}$.

Keywords—Silicon photomultiplier (SiPM), single-photon avalanche photodiode (SPAD), visible light communications (VLC), optical wireless communications $(\mathrm{OWC})$
\end{abstract}

\section{INTRODUCTION}

Visible light communications (VLC) is actively being investigated as a technology which could be used to augment WiFi in future heterogeneous networks. As with all communications systems the capacity of a VLC system depends upon its bandwidth and the signal to noise ratio (SNR) at the receiver output. One approach to improving the receiver SNR that is being investigated is to employ silicon photomultipliers (SiPMs) in receivers. These devices are arrays of single photon avalanche diodes (SPADs), with each SPAD known as a 'microcell', which can detect single photons. In VLC systems, this allows links to operate within a few photons per bit of the 'quantum' limit dictated by Poisson/shot noise. Consequently, a receiver incorporating a SiPM has been shown to be $9 \mathrm{~dB}$ more sensitive at $1 \mathrm{Gbps}$ than the best receiver based upon an avalanche photodiode[1]. However, after detecting a photon each microcell in a SiPM must be recharged. During this recharging process, which typically last several nanoseconds, a microcell is not able to detect photons and the response of SiPMs saturates[1].

The maximum rate of output pulses when a SiPM is saturated can be calculated easily using $\dot{C}_{\text {max }}=N / \tau e$ where $N$ is the number of microcells in the SiPM, $\tau$ is the microcell recharge time and $e$ is Euler's number. However, the corresponding data rate depends upon the number of detected photons required per bit which always depends upon the number of detected photons per bit from ambient light[1]. In addition, at date rates of more than $1 \mathrm{Gbit} / \mathrm{s}$ equalisation techniques are needed to overcome the intersymbol interference (ISI) caused by the limited bandwidth of the transmitter and the finite duration of the SiPM output pulses. These equalisation techniques require an increased signal to noise ratio which means that it is not currently possible to predict the maximum OOK data rate when the response of a SiPM is saturated. The relationship between the maximum rate at which output pulses can be generated by a SiPM and the maximum data rate has therefore been investigated.

\section{SIPM SATURATION}

The two SiPMs used in this investigation were both manufactured by ON Semiconductor[2]. One of these SiPMs was a J-Series 30035 which has 5,676 microcells, a recharge time of $45 \mathrm{~ns}$ and hence a maximum output pulse rate of $46.4 \times 10^{9}$ pulses/s. In contrast the J-Series 30020 has 14,410 microcells and a recharge time of $15 \mathrm{~ns}$. These parameters mean that its maximum output pulse rate is 7.6 times higher than the maximum pulse rate of the 30035 . Although they have very different maximum output pulse rates they have very similar output pulse widths. Any difference between their maximum data rates will therefore arise from very different output pulse rates in saturation.

After detecting a photon each microcell is recharged by the voltage source used to bias all the microcells above their breakdown voltage. Since each microcell has a capacitance that has to be recharged, the current flowing from the voltage source depends upon the rate at which photons are detected and hence output pulses generated. This means that a convenient method of observing the saturation of a SiPMs response is to measure the relationship between the irradiance falling onto the SiPM and the current provided by the voltage source biasing the SiPM[1]. Figure 1 shows the current supplied by this voltage source and the irradiance falling onto the 30020 and $30035 \mathrm{SiPMs}$. As expected, the current supplied to both SiPMs is proportional to the irradiance at low irradiances. However, the difference between their photon detection efficiencies and the capacitance per microcell means that the 30020 requires less current, and hence power, at a particular irradiance. This lower current per detected photon means that the similar maximum currents correspond to quite different maximum count rates. A comparison of the irradiances at which the measured response is $50 \%$ of the extrapolated linear response shows that the 30020 saturates at an irradiance which is six times higher than the irradiance at which the 30035 shows the same reduction in response.

\section{DATA RATES OBTAINED WITH DFE}

The experimental setup used to transmit data to the 30020 SiPM was the same as the setup used previously[1], except that a Mini Circuits ZX60-43S+ was used as the RF amplifier on the output of the SiPM. With a target bit error rate (BER) set at 10 ${ }^{-3}$ eight target data rates of interest were identified which were approximately equispaced between $500 \mathrm{Mbits} / \mathrm{s}$ and $3.45 \mathrm{Gbits} / \mathrm{s}$. At 
these data rates the finite width of the output pulses and other transient effects are expected to cause inter-symbol interference and so the BER was measured after decision feedback equalisation (DFE) had been employed. For each data rate the intensity of the transmitter was varied with a wire-grid polariser until the BER was approximately $10^{-3}$. To obtain a more accurate result the data rate was then varied until the data rate with the required BER was found. Finally, for each data rate the average irradiance of the transmitter at the SiPM was measured by replacing the SiPM with an 818-SL calibrated photodiode.

Figure 2 shows the data rates obtained at different irradiances when the BER is $10^{-3}$ and DFE had been employed. These results show that before either SiPM is saturated the slightly lower photon detection efficiency of the 30020 means that it requires a higher irradiance than the 30035 to achieve the same data rates. However, for data rates above $2 \mathrm{Gbits} / \mathrm{s}$, saturation of the 30035 means that the 30020 gives higher data rates at the same irradiance. The most unexpected feature of the results in Figure 2 is that, despite having a maximum count rate that is 7.6 times higher than the 30035 , the maximum data rate of the 30020 is only a factor of approximately 1.4 times higher than the 30035 . The data in Figure 2 at low data rates suggests that this is because the irradiance must increase by a factor of approximately 10 to increase the data rate by $1.5 \mathrm{Gbits} / \mathrm{s}$. The ratio of irradiances at which the SiPMs saturate would then suggest that the maximum data rate achievable by the 30020 should be $3.57 \mathrm{Gbits} / \mathrm{s}$, which is consistent with the results in Figure 2. These results therefore suggest that even higher data rates will be achieved if the transient response of both the transmitter and the SiPM can be improved.

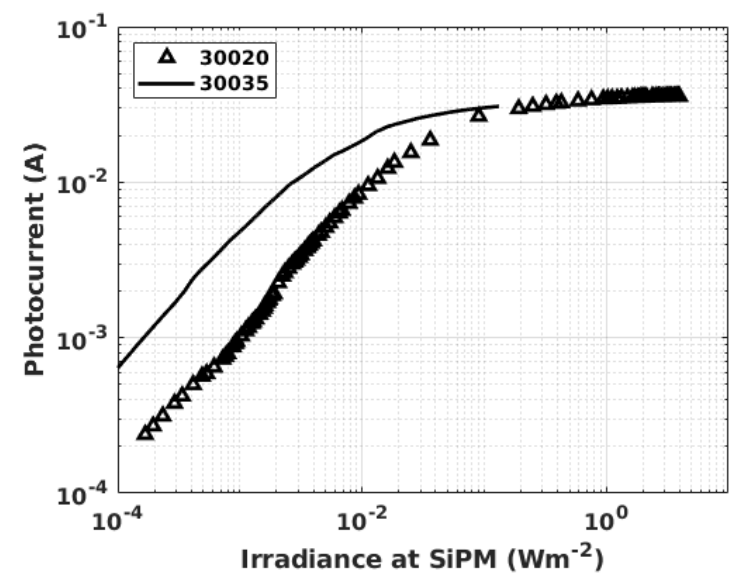

Figure 1 - Dynamic range of 30020 and 30035 when biased to $28 \mathrm{~V}$ and illuminated by a $405 \mathrm{~nm}$ laser.

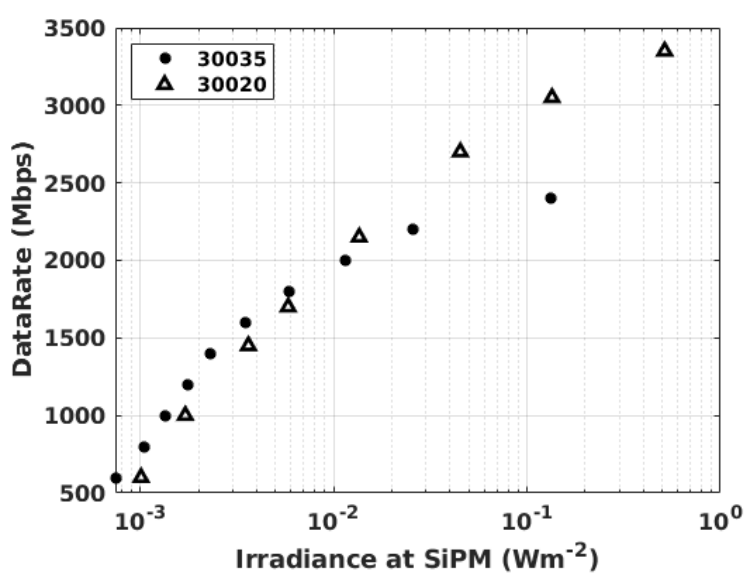

Figure 2 - Results obtained for VLC links using OOK and DFE targeting a BER of $10^{-3}$.

\section{CONCLUSION}

Recently, it was reported that despite saturation when a 30035 SiPM is used as a receiver the maximum OOK data rate with a BER of $10^{-3}$ was $2.4 \mathrm{Gbits} / \mathrm{s}$. Results have been presented which show, that because it has a higher maximum output pulse rate, a $30020 \mathrm{SiPM}$ can achieve the same BER at a data rate of $3.45 \mathrm{Gbits} / \mathrm{s}$. This is a new record for the data rate achieved with a SiPM receiver. Unfortunately, saturation means that these high data rates occur at irradiances of more than $100 \mathrm{mWm}^{-2}$. At irradiances which are more likely to occur when a VLC system is deployed either SiPM can support a data rate of up to 2 Gbits/s. In these situations the higher PDE of the 30035 means that it achieves a slightly higher data rate at the same irradiance than the 30020 . However, at the same irradiance the 30020 consumes only a fraction of the power of the 30035 . The 30020 may therefore be more suitable for systems, such as a smart phone, where power consumption is an important factor. In the future it should be possible to achieve even higher maximum data rates by using SiPMs with narrower output pulses.

\section{REFERENCES}

[1] Z. Ahmed, R. Singh, W. Ali, G. Faulkner, D. O’Brien and S. Collins, "A SiPM-Based VLC Receiver for Gigabit Communication Using OOK Modulation," in IEEE Photonics Technology Letters, vol. 32, no. 6, pp. 317-320, 15 March15, 2020

[2] Onsemi.com. 2020. J-Series SiPM Sensors Datasheet. [online] Available at: https://www.onsemi.com/pub/Collateral/MICROJ-SERIES-D.PDF, Accessed 10 March 2020.

This work has been partly supported by the Punjab Educational Endowment Fund, Pakistan and by the UK Engineering and Physical Sciences Research Council (EPSRC) under Grant EP/R00689X/1. 